\title{
Heat shock protein complex vaccines induce antibodies against Neisseria meningitidis via a MyD88-independent mechanism
}

\author{
Jia-Xi Han ${ }^{1,2}$, Garrett Z Ng ${ }^{1,2}$, Paola Cecchini ${ }^{3}$, Yok Teng Chionh ${ }^{1,2}$, Muhammad A \\ Saeed $^{1,2}$, Lisbeth M. Næss ${ }^{4}$, Michael Joachim, ${ }^{3}$ Lucy E Blandford ${ }^{3}$ Richard A \\ Strugnell $^{4 \underline{5}}$, Camilo A Colaco ${ }^{3}, \&$ Philip Sutton ${ }^{1,2 \#}$
}

1 Mucosal Immunology, Murdoch Childrens Research Institute, Royal Children's Hospital, Melbourne, Parkville, VIC 3052, Australia

2 Centre for Animal Biotechnology, Faculty of Veterinary and Agricultural Science, The University of Melbourne, Parkville, VIC 3010, Australia

3 ImmunoBiology Ltd., Babraham Research Campus, Babraham, Cambridge, UK 4 Division of Infectious Disease Control, Norwegian Institute of Public Health, Oslo, Norway

54 Department of Microbiology and Immunology, University of Melbourne at The Peter Doherty Institute for Infection and Immunity, Parkville, Victoria 3010, Australia

\#Author for Correspondence:

Tel: +61-3-9936-6751; Fax: +61-3-9348-1391

Email: phil.sutton@mcri.edu.au 


\section{ABSTRACT}

\section{BACKGROUND:}

Neisseria meningitidis are common colonisers of the human nasopharynx. In some circumstances, $N$. meningitidis becomes an opportunistic pathogen that invades tissues and causes meningitis. While a vaccine against a number of serogroups has been in effective use for many years, a vaccine against $N$. meningitidis group B has not yet been universally adopted. Bacterial Heat shock protein complex (HSPC) vaccines comprise bacterial HSPs, purified with their chaperoned protein cargo. HSPC vaccines use the intrinsic adjuvant activity of their Hsp, thought to act via Tolllike receptors (TLR), to induce an immune response against their cargo antigens. This study evaluated $\mathrm{HspC}$ vaccines from $N$. meningitidis and the closely related commensal N. lactamica.

\section{RESULTS:}

The protein composition of $N$. lactamica and $N$. meningitidis HSPCs were similar. Using human HEK-293 cells we found that both HSPCs can induce an innate immune response via activation of TLR2. However stimulation of TLR2 or TLR4 deficient murine splenocytes revealed that HSPCs can activate an innate immune response via multiple receptors. Vaccination of wildtype mice with the Neisseria HSPC induced a strong antibody response and a Th1-restricted $\mathrm{T}$ helper response. However, vaccination of mice deficient in the major TLR adaptor protein, MyD88, revealed that while the Th1 response to Neisseria HSPC requires MyD88, these vaccines unexpectedly induced an antigen-specific antibody response via a MyD88independent mechanism.

\section{CONCLUSIONS:}

$N$. lactamica and N. meningitidis HSPC vaccines both have potential utility for immunising against neisserial meningitis without the requirement for an exogenous adjuvant. The mode of action of these vaccines is highly complex, with HSPCs inducing immune responses via both MyD88-dependent and -independent mechanisms. In particular, these HSPC vaccines induced an antibody response without detectable T cell help.

Keywords: Heat shock protein, Heat shock protein complex vaccine, Neisseria meningitidis, meningitis, Neisseria lactamica 


\section{INTRODUCTION}

Neisseria is a large genus of gram-negative, aerobic diplococcal bacteria that colonize various mucosal surfaces in a range of animals. The majority of Neisseria species are typically harmless commensals, such as $N$. lactamica which colonize the human nasopharynx [1]. A few however are significant pathogens, including $N$. meningitidis which also occupies the same nasal niche. $N$. meningitidis is a common coloniser of humans, with up to $10 \%$ of individuals carrying this organism with no illeffect. In a small proportion of those infected however, and for reasons that are poorly understood, $N$. meningitidis can turn into an opportunistic pathogen invading the tissues and disseminating to become a major cause of meningitis or septicaemia [2]. The pathogenic effects of $N$. meningitidis infection are far more prevalent in children.

$N$. meningitidis is separated into serogroups based on its polysaccharide capsule; 13 have been identified with $\mathrm{A}, \mathrm{B}, \mathrm{C}, \mathrm{X}, \mathrm{W} 135$ and $\mathrm{Y}$ being those most commonly associated with disease [3]. A highly successful vaccine that induces antibodies against many of these major serogroup polysaccharides has been available for over ten years, having a major impact on reducing meningococcal disease [4]. Due to similarities between $N$. meningitidis type B and human polysaccharides, it was not possible to develop a similar vaccine against this serogroup. An aluminium hydroxide adjuvanted protein-based type B vaccine recently become available [5, 6], although this vaccine has not yet been universally adopted internationally. There thus remains a need for an effective and economical vaccine against $N$. meningitidis to protect against meningococcal disease.

Proteomic, genetic and immunological analyses have indicated considerable similarity between $N$. lactamica and $N$. meningitidis antigenicity [7-9], and there is evidence that $N$. lactamica colonization can provide some protection against $N$. meningitidis infection [10], possibly either by competing for colonization sites or via the induction of cross-reactive antibodies [11]. This has resulted in N. lactamica being studied as a potential vaccine tool for inducing protective immunity against $N$. meningitidis [9, 12-14].

Heat shock proteins (HSPs) are highly conserved molecules, grouped into families, mostly based on their molecular weight and sequence homology. Main family members include HSP90, HSP70 and HSP60 in mammalian cells, while in bacteria HSP60 (also called GroEL) and HSP70 (DnaK) are generally the most prevalent forms. The predominant role of HSPs is to serve as chaperones, binding and providing 
stability to cytosolic proteins, particularly during folding and unfolding. However HSPs also serve a number of other functions, including the ability to act as a link between the innate and acquired immune systems, for example by delivering antigens for immune presentation and acting as damage associated molecular pattern (DAMP) molecules that can activate Toll-Like receptors (TLR) [15]. HSP-induced innate immune signalling has been particularly linked with the activation of TLR2 and TLR4 [16].

The enrichment of HSP from bacteria along with its bound protein cargo (termed the HSP Complex; HSPC) provides a source of immunogen that, because of the intrinsic immunostimulatory activity of the HSP and the range of antigenic molecules it is associated with, can potentially produce an effective vaccine without requiring the addition of an exogenous adjuvant [17-19].

While the mechanism of action of such non-exogenously adjuvanted HSPC vaccine formulations was predicted to be via HSP activation of TLR2 and/or TLR4, this has not previously been experimentally confirmed. In this study, we characterize and compare the antigenic cargo of HSPC from closely-related commensal ( $N$. lactamica) and pathogenic (N. meningitidis) nasal-colonizing Neisseria species and use these formulations to examine the importance of TLR activation in the immune response to HSPC vaccination.

\section{MATERIALS AND METHODS}

\section{Preparation of Neisseria HSPC}

HSPCs from $N$. lactamica strain Y92/1009 or $N$. meningitidis strain H44/76 were prepared essentially as described [20]. Lysed bacteria (using a Triton X-100 solution; $40 \mathrm{mM}$ Tris, $1 \mathrm{mM} \mathrm{MgCl}_{2}, 20 \mathrm{mM} \mathrm{NaCl}, 0.5 \%$ Triton X-100, $\mathrm{pH} 8.0$ ), were centrifuged at 13,000 rpm, passed through a $0.2 \mu \mathrm{m}$ filter, and $10 \mathrm{mg}$ of clarified lysate loaded onto a 5mL ion exchange column (Capto Q, GE Healthcare, Uppsala) at $0.5 \mathrm{~mL} / \mathrm{min}$. HSPCs were eluted using a high salt buffer $(40 \mathrm{mM}$ Tris- $\mathrm{HCl} \mathrm{pH} 8.0$ containing $300 \mathrm{mM} \mathrm{NaCl}$ and $0.5 \%$ Triton X-100). Fractions were analysed for protein concentration (Bradford assay) and HSP60 content by western blot using the anti-HSP60 antibody SPA-875 (Stressgen Bioreagents, Ann Arbor, MI, USA). The final vaccine comprised pooled fractions containing most HSP60, sterile filtered (0.2 
$\mu \mathrm{m}$ filter) and diluted to $500 \mu \mathrm{g} / \mathrm{mL}$ in $\mathrm{pH} 8.0$ buffer containing $40 \mathrm{mM}$ Tris, $1 \mathrm{mM}$ $\mathrm{MgCl}_{2}, 300 \mathrm{mM} \mathrm{NaCl}$ and $0.1 \%$ Triton X-100.

The reduction of contaminating Neisserial lipopolysaccharide (LPS) from $N$. lactamica HSPCs was performed using an EndoTrap ${ }^{\circledR}$ HD kit (hyglos, Charleston, USA). LPS levels in the HSPCs used in this study, as quantified by Endosafe®PTS $^{\text {TM }}$ (Charles River, Wilmington, Mass, USA), were determined to be 14,155 ( $N$. lactamica HSPC), 66 (N. lactamica HSPC LPS-reduced) and 19,679 ( $N$. meningitidis HSPC) EU/mg.

\section{Proteomic analysis of Neisseria HSPC}

For proteomic analysis, proteins were denatured in urea (Life Technologies, Carlsbad, USA), reduced with 10 mM TCEP (Pierce, Rockford, IL, USA), alkylated with $55 \mathrm{mM}$ iodoacetamide (Sigma-Aldrich, St. Louis, USA) and digested with sequencing grade modified trypsin (Thermo Scientific Pierce) overnight at $37^{\circ} \mathrm{C}$. Peptides were acidified with $1 \%$ formic acid (Sigma-Aldrich), purified through solid phase extraction with Oasis HLB cartridges (Waters, Milford, USA) and freeze-dried before analysis by liquid chromatography-tandem mass spectrometry (LC-MSMS) as previously described [17]. Data analysis was carried out using Proteome Discoverer (Thermo Scientific version 1.4) with the Mascot search engine (Matrix Science version 2.4) against the Uniprot database. Search results were set to a maximum of $1 \%$ false discovery rate (FDR), one missed trypsin cleavage, and text-filtered for Neisseria. Proteins were positively identified if they contained at least two unique peptides in at least two of three sample replicates. Percentage molarity was calculated based on the exponentially modified protein abundance index [21].

For protein visualization, $5 \mu \mathrm{g}$ HSPC was compared with $5 \mu \mathrm{g}$ lysate on Coomassie Blue stained SDS-PAGE gels as previously described [22].

\section{Cell stimulation assays}

HEK293 cells and splenocytes from C57BL/6, $T l r 2^{-/-}$or $T l r 4^{0 / 0}$ mice $[23,24]$, were cultured in DMEM (high glucose) or RPMI 1640 respectively both containing 10\% foetal calf serum, penicillin, streptomycin, glutamine (Life Technologies) and 2.5 $\mu \mathrm{g} / \mathrm{mL}$ amphotericin B (Sigma-Aldrich). HEK293 cells expressing TLR2 [25], were kindly provided by Dr Ashley Mansell (Monash Institute of Medical Research). Cells were stimulated with $5 \mu \mathrm{g} / \mathrm{mL}$. lactamica lysate or Neisseria HSPC vaccine, while controls were cultured in media alone, or stimulated with the TLR4 ligand ultrapure 
LPS at $100 \mathrm{ng} / \mathrm{mL}$, or the TLR2 ligand Pam3CysSerLys4 (P3C) at $50 \mathrm{ng} / \mathrm{mL}$ (Invivogen, San Diego, CA, USA).

\section{Evaluation of cytokine levels}

Cytokine concentrations were determined by enzyme linked immunosorbent assay (ELISA). 96-well Maxisorp plates (Nunc, Roskilde, Denmark) were coated with purified anti-mouse MIP2 (100 ng/well), IL-10 (200 ng/well), TNFa (40 ng/well)(R\&D Systems, Minneapolis, MN, USA), IL-6, IL-13, IL-17A (50 ng/well)(eBioscience, San Diego, CA, USA), IFN $\gamma$ (100 ng/well)(BD Biosciences, San Jose, CA, USA), or anti-human IL-8 (200 ng/well)(R\&D Systems) at $4^{\circ} \mathrm{C}$ overnight in bicarbonate coating buffer, $\mathrm{pH}$ 9.6. Plates were blocked with $1 \%$ BSA (Sigma-Aldrich) in PBS (blocker buffer) for one hour prior to addition of samples in duplicate at $4{ }^{\circ} \mathrm{C}$ overnight. Captured cytokines were then labelled with biotinylated anti-mouse MIP2 (3.9 ng/well), IL-10 (2.5 ng/well), TNF $\alpha$ (15 ng/well)(R\&D Systems), IL-6 (25ng/well), IL-13 (25 ng/well) IL-17A (25 ng/well)(eBioscience), IFN $\gamma(50 \mathrm{ng} /$ well)(BD Biosciences) or anti-human IL-8 (1 ng/well)(R\&D Systems) in blocker buffer for one hour prior to the addition of $50 \mu \mathrm{L}$ horseradish peroxidase conjugated streptavidin (Pierce) 1/5000 in blocker buffer for $30 \mathrm{~min}$. Colour was developed with $100 \mu \mathrm{L}$ of TMB solution prepared as $1 \%$ of $10 \mathrm{mg} / \mathrm{mL}$ TMB (SigmaAldrich) in DMSO and $0.006 \%$ hydrogen peroxide in phosphate-citrate buffer, $\mathrm{pH}$ 5.0 , and the reaction stopped with an equal volume of $2 \mathrm{M}$ sulphuric acid prior to reading absorbance at $450 \mathrm{~nm}$. Sample concentration was determined against a standard curve of recombinant cytokine (same manufacturers as antibodies).

\section{Mouse vaccination experiments}

Animal experimentation was performed under institutional guidelines and with approval from the University of Melbourne Animal Ethics Committee. Groups of agematched, C57BL/6 or $M y d 88^{-/}$mice (Walter and Eliza Hall Institute, Melbourne, Australia) [26], were injected subcutaneously with either $100 \mu \mathrm{g}$ of HSPC (vaccinated) or an equal volume of sterile phosphate buffered saline (controls). Mice were vaccinated twice, four weeks apart, then euthanized and tissues collected for analysis one week after the second immunization.

\section{Evaluation of serum antibody responses}

Blood was collected by cardiac puncture and left to coagulate prior to collection of sera. Levels of specific antibodies were quantified by ELISA. Maxisorp 
immunoplates (Nunc) were coated with $5 \mu \mathrm{g} /$ well of $N$. lactamica lysate at $4{ }^{\circ} \mathrm{C}$ overnight in bicarbonate coating buffer, $\mathrm{pH}$ 9.6. Plates were blocked for one hour at room temperature then 1/20 serially diluted sera in blocker added in duplicate for one hour at room temperature. Captured antibodies were labelled with 4 ng/well horseradish peroxidase-conjugated goat anti-mouse immunoglobulin G (IgG) (Pierce), 8 ng/well horseradish peroxidase-conjugated goat anti-mouse IgG1 (Southern Biotech, Birmingham, AL, USA) or $10 \mathrm{ng} /$ well horseradish peroxidase-conjugated goat anti-mouse IgG2c (Immunology Consultants Laboratory, Portland, OR, USA) in blocker for one hour. Colour was developed and read as above prior to calculation of endpoint titres. Serum bactericidal activity against $N$. meningitidis was performed as previously described [27].

\section{Statistics}

For statistical analyses, all data were log-transformed then compared by analysis of variance (ANOVA) with Dunnett's post-hoc analysis, using SPSS software, version 21.0.

\section{RESULTS}

\section{Characterization of Neisseria lactamica HSPC}

$N$. lactamica and N. meningitidis HSPCs were made using an optimized and standard procedure [20], originally developed to produce and analyse HSPCs from other bacteria [17]. The composition of $N$. lactamica and $N$. meningitidis HSPCs were examined by proteomic analysis and the twenty most abundant proteins are listed in Table 1. The antigenic compositions of the HSPCs from these two nasal Neisseria species were notably similar. In addition to the expected HSPs (HSP60 and HSP70), many of the most highly abundant Neisseria HSPC components were ribosomeassociated proteins.

TLR2 and TLR4 signalling in the cytokine response to $N$. lactamica and $N$ meningitidis HSPC

Some HSPs induce an innate immune response by activation of TLR2. We therefore examined whether Neisseria HSPC were also capable of stimulating a response via this receptor. Culture of HEK-293 cells that express TLR2 with HSPC from $N$. lactamica or $N$. meningitidis induced the secretion of IL-8, in contrast to control 
HEK-293 cells which were unresponsive to both HSPC and TLR2 activation (Figure 1). No response to LPS was observed for any cells, demonstrating that the HSPC induced secretion of IL-8 was not related to contaminating endotoxin. Hence HSPC from both Neisserial species could trigger an innate immune response via TLR2.

To extend this observation, as other HSP have been shown to activate both TLR2 and TLR4, we stimulated splenocytes from wildtype, $T l r 2^{-/}$and $T l r 4^{0 / 0}$ mice with $N$. lactamica or N. meningitidis HSPC. While neither HSPC induced secretion of antiinflammatory IL-10, both induced the secretion of innate proinflammatory cytokines (IL-6, MIP2, TNF $\alpha$ ) equivalent to or greater than the levels induced by the positive controls (LPS for $T l r 2^{-/}$cells and P3C for $T l r 4^{0 / 0}$ cells) (Figure 2). It was notable that the cytokine response of $T l r 4^{0 / 0}$ cells to $N$. lactamica HSPC was significantly greater than that induced by $N$. meningitidis HSPC, suggesting a possibly greater role for TLR4 activation in the response to $N$. meningitidis HSPC.

Hence these data indicated that Neisseria HSPC stimulated the secretion of innate cytokines via a mechanism that was not solely dependent on either TLR2 or TLR4. This raised the possibility that these HSPCs were activating both TLR2 and TLR4, although signalling via TLR4 might play a larger role in the response induced by $N$. meningitidis HSPC.

\section{MyD88-dependent pathways in the response to Neisseria HSPC vaccination}

As our data suggested that Neisseria HSPC might activate multiple TLR pathways, we vaccinated mice lacking $\operatorname{MyD} 88\left(\right.$ Myd88 $\left.^{--}\right)$with HSPC from $N$. lactamica. MyD88 is an adaptor protein essential for most TLR-dependent signalling pathways. In addition, to determine whether contaminating LPS may contribute to the functionality of such a vaccine, we also immunized an additional group of mice with $N$. lactamica HSPC depleted of $99.5 \%$ of the endotoxin by repeated passage over an EndoTrap $^{\circledR}$ HD resin column. One dimensional SDS-PAGE comparisons demonstrated that LPS depletion had no effect on the antigenic composition of $N$. lactamica HSPC (Figure 3a).

Serum antibodies play a critical role in the protective response to pathogenic Neisserial infection $[4,28]$. Vaccination with $N$. lactamica HSPC induced a large antigen-specific serum IgG antibody response in both wildtype and $M y d 88^{-/}$mice against both $N$. lactamica and $N$ meningitidis (Figure $3 b$ ). The levels of this antibody 
response in these two strains of mice was indistinguishable, indicating the serum $\operatorname{IgG}$ response to $N$. lactamica HSPC vaccine occurred independently of MyD88-signalling.

A substantial Neisseria-specific serum IgG response also developed in mice receiving the LPS-reduced vaccine, although in the response to $N$. lactamica, this was significantly lower in mice receiving HSPC containing endotoxin (Figure 3b). This suggests the antibody response was partially, though not entirely, adjuvanted by LPS present in the formulation. As this effect was present in both wildtype and $M y d 88^{-/-}$ mice, any effect of LPS on the resulting antibody response occurred via a MyD88independent mechanism.

To examine the adaptive $\mathrm{T}$ cell memory response induced by these vaccinations, splenocytes from these mice were stimulated in vitro with $N$. lactamica lysate. Vaccination with $N$. lactamica HSPC induced a strong memory Th1-type response as demonstrated by significant secretion of IFN $\gamma$ by antigen-stimulated spleen cells from immunized wildtype mice (Figure 3c). A similar level of response was observed in cells from mice vaccinated with the LPS-depleted formulation. Furthermore, in contrast to the antibody response, induction of the IFN $\gamma$ recall response was completely dependent on MyD88 as no IFN $\gamma$ was secreted by antigen-stimulated

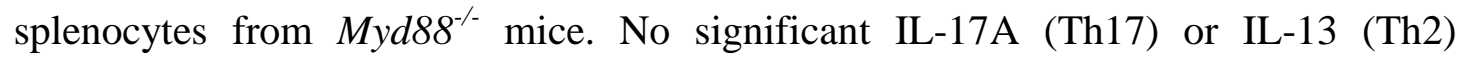
secretion was induced by $N$. lactamica stimulation of splenocytes from any group (Figure 3c).

This experiment was repeated using $N$. meningitidis HSPC with similar results. Vaccination of wildtype mice with $N$. meningitidis HSPC also induced a very strong serum IgG antibody response. We thus quantified the IgG subclasses and found that the antibody response comprised both $\mathrm{IgG} 1$ and $\operatorname{IgG} 2 \mathrm{c}$ and, as for $N$. lactamica HSPC, was not significantly different between wildtype and $M y d 88^{-/}$mice (Figure 4a).

Stimulation of splenocytes from these vaccinated mice with $N$. meningitidis HSPC induced a MyD88-dependent IFN $\gamma$ response, without either IL-17A or IL-13 production (Figure 4b), as seen with $N$. lactamica $\mathrm{HSPC}$ vaccination. In contrast to $N$. lactamica HSPC however, this occurred as a basal response of naïve cells, with no evidence of a Th1-type memory response, as shown by a lack of increased IFN $\gamma$ secretion by splenocytes from $N$. meningitidis HSPC vaccinated, as compared to naïve (unvaccinated) animals (Figure 4b). 
These observations indicate that the antibody and Th1 cellular response to Neisseria HSPC vaccination were induced by two distinct mechanisms, which were MyD88independent and dependent respectively.

\section{DISCUSSION}

While $N$. lactamica and $N$. meningitidis are related bacterial species that occupy the same niche in their human host, there is a major difference in their ability to cause disease. A number of studies have compared these organisms seeking to explain this difference in pathogenicity. At the genetic level there is some uncertainty as to whether commensal $N$. lactamica and pathogenic $N$. meningitidis differ greatly with respect to their expression of virulence factors [8, 29, 30]. Several previous studies have also compared the proteomes of these bacteria, however such studies have typically focused on their outer membrane components, motivated predominantly as strategies to identify candidate vaccine antigens $[7,31]$.

This study provides the first analysis and comparison of the HSPC, the chaperoned proteins associated with the heat shock proteins, of $N$. lactamica and $N$. meningitidis as vaccine candidates. Proteomic analysis revealed no obvious major differences between the composition of HSPC from commensal $N$. lactamica and HSPC from the pathogen $N$. meningitidis $\mathrm{B}$. While only a crude comparison is possible, this does not point to any clear features that contribute to pathogenicity. This antigenic similarity importantly supports the utility of HSPCs from either N. lactamica or N. meningitidis in a meningitis vaccine. Furthermore, HSPC from both $N$. lactamica and $N$. meningitidis were shown to be capable of 1) activating cells via TLR2-dependent signalling in the human HEK293 cell line, and 2) inducing cytokine secretion by mouse immune cell, via a mechanism that requires MyD88 but is not entirely dependent on either TLR2 or TLR4. This is consistent with HSPC signalling via multiple innate receptors including both TLR2 and TLR4. It is well documented that Neisseria can induce TLR2-signalling via their porin PorB [32-35]. However our proteomic analysis revealed a complete absence of porins in both HSPC, indicating TLR2 activation by these vaccines did not involve PorB.

It was interesting to note that the mouse cytokine response to $N$. lactamica HSPC was at least equivalent to that induced by $N$. meningitidis HSPC. This contrasts with the poor immune response to $N$. lactamica, relative to $N$. meningitidis, that has been reported in studies using whole bacteria $[36,37]$. Hence the poor immunogenicity of 
$N$. lactamica does not appear to be related to a lower immune-activating potential of their HSPs. HSPCs from both Neisserial species induced a Th1 but not a Th2 or Th17 response and such a Th1-polarizing effect has previously also been reported for HSP from both humans and other bacteria [38, 39]. In contrast, TLR4-deficient cells stimulated with $N$. meningitidis and $N$. lactamica HSPC secreted different levels of innate cytokines. This indicates important differences in the pathways by which individual HSPCs, even from closely related bacteria, activate an immune response with TLR4 appearing to play a greater role in the response to N. meningitidis HSPC.

Protective immunity against $N$. meningitidis is believed to be antibody mediated. While an analysis of the bactericidal activity of this response was beyond the aims of

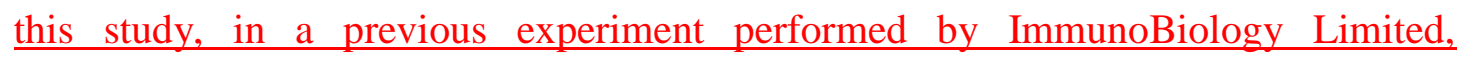
subcutaneous vaccination of CD1 mice $(\mathrm{n}=10)$ with $50 \mu \mathrm{g}$ of $N$. meningitidis HSPC induced serum bactericidal activity (titre >1:512) against the homologous H44/76 strain, in contrast to no activity in sera from sham-vaccinated controls. Tand the ability of HSPs to activate TLRs is a key mechanism by which HSPC induce an immune response to co-delivered antigens [18]. An important and surprising finding of the current study therefore, was that Neisseria HSPC potently induced an effective antibody response, even without addition of an exogenous adjuvant, in MyD88deficient mice. This result was unexpected and indicates these HSPC vaccines induce a humoral response via a currently unidentified MyD88-independent mechanism. Perhaps related to this mechanism are recent demonstrations that MyD88 is also not essential for the generation of an antibody response against adenoviral capsid protein and bacterial flagellin [40, 41].

In conclusion, the type and similarity of the immune response mounted against vaccination with $N$. lactamica and $N$. meningitidis HSPC, both with respect to cellmediated immunity and in the production of a specific antibody response, suggests that either formulation has potential utility in a meningococcal vaccine. Our examination of the mode of action of these vaccines has revealed a high level of complexity, with HSPC inducing immune responses via a number of mechanisms, including a MyD88-dependent cellular response with subtle differences in the use of innate receptors, and a MyD88-independent mechanism that induces an antibody response without any detectable $\mathrm{T}$ helper cell response. Further studies are required to identify the mechanism by which HSPC induce this apparently $\mathrm{T}$ helper cellindependent antibody response. 


\section{ACKNOWLEDGEMENTS}

This work was supported by the Victorian Government's Operational Infrastructure Support Program, ARC Linkage Grant LP120100226 from the Australian Research Council and ImmunoBiology Limited. PS is supported by a Senior Research Fellowship from the National Health and Medical Research Council of Australia.

\section{REFERENCES}

[1] Olsen SF, Djurhuus B, Rasmussen K, Joensen HD, Larsen SO, Zoffman H, et al. Pharyngeal carriage of Neisseria meningitidis and Neisseria lactamica in households with infants within areas with high and low incidences of meningococcal disease. Epidemiology and infection. 1991;106:445-57.

[2] Miller F, Lecuyer H, Join-Lambert O, Bourdoulous S, Marullo S, Nassif X, et al. Neisseria meningitidis colonization of the brain endothelium and cerebrospinal fluid invasion. Cellular microbiology. 2012.

[3] Read RC. Neisseria meningitidis; clones, carriage, and disease. Clinical microbiology and infection : the official publication of the European Society of Clinical Microbiology and Infectious Diseases. 2014;20:391-5.

[4] McIntyre PB, O'Brien KL, Greenwood B, van de Beek D. Effect of vaccines on bacterial meningitis worldwide. Lancet. 2012;380:1703-11.

[5] Serruto D, Bottomley MJ, Ram S, Giuliani MM, Rappuoli R. The new multicomponent vaccine against meningococcal serogroup $\mathrm{B}$, 4CMenB: immunological, functional and structural characterization of the antigens. Vaccine. 2012;30 Suppl 2:B87-97.

[6] Andrews SM, Pollard AJ. A vaccine against serogroup B Neisseria meningitidis: dealing with uncertainty. The Lancet infectious diseases. 2014;14:426-34.

[7] Abel A, Sanchez S, Arenas J, Criado MT, Ferreiros CM. Bioinformatic analysis of outer membrane proteome of Neisseria meningitidis and Neisseria lactamica. International microbiology : the official journal of the Spanish Society for Microbiology. 2007;10:5-11.

[8] Snyder LA, Saunders NJ. The majority of genes in the pathogenic Neisseria species are present in non-pathogenic Neisseria lactamica, including those designated as 'virulence genes'. BMC genomics. 2006;7:128. 
[9] Troncoso G, Sanchez S, Criado MT, Ferreiros CM. Analysis of Neisseria lactamica antigens putatively implicated in acquisition of natural immunity to Neisseria meningitidis. FEMS immunology and medical microbiology. 2002;34:9-15. [10] Oliver KJ, Reddin KM, Bracegirdle P, Hudson MJ, Borrow R, Feavers IM, et al. Neisseria lactamica protects against experimental meningococcal infection. Infection and immunity. 2002;70:3621-6.

[11] Andrade JR, Marques Mda C, de Santa Rosa MR. Nasal secretions of Neisseria lactamica carriers have an inhibitory effect on Neisseria meningitidis attachment to human oroepithelial cells. Memorias do Instituto Oswaldo Cruz. 1986;81:453-7.

[12] Gorringe AR, Taylor S, Brookes C, Matheson M, Finney M, Kerr M, et al. Phase I safety and immunogenicity study of a candidate meningococcal disease vaccine based on Neisseria lactamica outer membrane vesicles. Clinical and vaccine immunology : CVI. 2009;16:1113-20.

[13] Li Y, Zhang Q, Winterbotham M, Mowe E, Gorringe A, Tang CM. Immunization with live Neisseria lactamica protects mice against meningococcal challenge and can elicit serum bactericidal antibodies. Infection and immunity. 2006;74:6348-55.

[14] Sanchez S, Troncoso G, Criado MT, Ferreiros C. In vitro induction of memorydriven responses against Neisseria meningitidis by priming with Neisseria lactamica. Vaccine. 2002;20:2957-63.

[15] Osterloh A, Breloer M. Heat shock proteins: linking danger and pathogen recognition. Medical microbiology and immunology. 2008;197:1-8.

[16] Colaco CA, Bailey CR, Walker KB, Keeble J. Heat shock proteins: stimulators of innate and acquired immunity. Biomed Res Int. 2013;2013:461230.

[17] Chionh YT, Arulmuruganar A, Venditti E, Ng GZ, Han JX, Entwisle C, et al. Heat shock protein complex vaccination induces protection against Helicobacter pylori without exogenous adjuvant. Vaccine. 2014;32:2350-8.

[18] McNulty S, Colaco CA, Blandford LE, Bailey CR, Baschieri S, Todryk S. Heatshock proteins as dendritic cell-targeting vaccines--getting warmer. Immunology. 2013;139:407-15.

[19] Colaco CA, Bailey CR, Keeble J, Walker KB. BCG (Bacille Calmette-Guerin) HspCs (heat-shock protein-peptide complexes) induce T-helper 1 responses and protect against live challenge in a murine aerosol challenge model of pulmonary tuberculosis. Biochemical Society transactions. 2004;32:626-8. 
[20] Colaco C, Salim K. Method for purifying protein complexes. 2010.

[21] Ishihama Y, Oda Y, Tabata T, Sato T, Nagasu T, Rappsilber J, et al. Exponentially modified protein abundance index (emPAI) for estimation of absolute protein amount in proteomics by the number of sequenced peptides per protein. Molecular \& cellular proteomics : MCP. 2005;4:1265-72.

[22] Cleveland DW, Fischer SG, Kirschner MW, Laemmli UK. Peptide mapping by limited proteolysis in sodium dodecyl sulfate and analysis by gel electrophoresis. The Journal of biological chemistry. 1977;252:1102-6.

[23] Kupz A, Guarda G, Gebhardt T, Sander LE, Short KR, Diavatopoulos DA, et al. NLRC4 inflammasomes in dendritic cells regulate noncognate effector function by memory CD8(+) T cells. Nature immunology. 2012;13:162-9.

[24] Takeuchi O, Hoshino K, Kawai T, Sanjo H, Takada H, Ogawa T, et al. Differential roles of TLR2 and TLR4 in recognition of gram-negative and grampositive bacterial cell wall components. Immunity. 1999;11:443-51.

[25] Latz E, Visintin A, Lien E, Fitzgerald KA, Monks BG, Kurt-Jones EA, et al. Lipopolysaccharide rapidly traffics to and from the Golgi apparatus with the toll-like receptor 4-MD-2-CD14 complex in a process that is distinct from the initiation of signal transduction. The Journal of biological chemistry. 2002;277:47834-43.

[26] Kawai T, Adachi O, Ogawa T, Takeda K, Akira S. Unresponsiveness of MyD88deficient mice to endotoxin. Immunity. 1999;11:115-22.

[27] Sandbu S, Feiring B, Oster P, Helland OS, Bakke HS, Naess LM, et al. Immunogenicity and safety of a combination of two serogroup B meningococcal outer membrane vesicle vaccines. Clinical and vaccine immunology : CVI. 2007;14:1062-9. [28] Jarvis GA. Recognition and control of neisserial infection by antibody and complement. Trends in microbiology. 1995;3:198-201.

[29] Stabler R, Hinds J. The majority of genes in the pathogenic Neisseria species are present in non-pathogenic Neisseria lactamica, including those designated as virulence genes: response. BMC genomics. 2006;7:129.

[30] Stabler RA, Marsden GL, Witney AA, Li Y, Bentley SD, Tang CM, et al. Identification of pathogen-specific genes through microarray analysis of pathogenic and commensal Neisseria species. Microbiology. 2005;151:2907-22.

[31] Vaughan TE, Skipp PJ, O'Connor CD, Hudson MJ, Vipond R, Elmore MJ, et al. Proteomic analysis of Neisseria lactamica and Neisseria meningitidis outer membrane vesicle vaccine antigens. Vaccine. 2006;24:5277-93. 
[32] Platt A, MacLeod H, Massari P, Liu X, Wetzler L. In vivo and in vitro characterization of the immune stimulating activity of the neisserial porin PorB. PloS one. 2013;8:e82171.

[33] Chiavolini D, Weir S, Murphy JR, Wetzler LM. Neisseria meningitidis PorB, a Toll-like receptor 2 ligand, improves the capacity of Francisella tularensis lipopolysaccharide to protect mice against experimental tularemia. Clinical and vaccine immunology : CVI. 2008;15:1322-9.

[34] Liu X, Wetzler LM, Nascimento LO, Massari P. Human airway epithelial cell responses to Neisseria lactamica and purified porin via Toll-like receptor 2-dependent signaling. Infection and immunity. 2010;78:5314-23.

[35] Liu X, Wetzler LM, Massari P. The PorB porin from commensal Neisseria lactamica induces Th1 and Th2 immune responses to ovalbumin in mice and is a potential immune adjuvant. Vaccine. 2008;26:786-96.

[36] Vaughan AT, Gorringe A, Davenport V, Williams NA, Heyderman RS. Absence of mucosal immunity in the human upper respiratory tract to the commensal bacteria Neisseria lactamica but not pathogenic Neisseria meningitidis during the peak age of nasopharyngeal carriage. J Immunol. 2009;182:2231-40.

[37] Fowler MI, Yin KY, Humphries HE, Heckels JE, Christodoulides M. Comparison of the inflammatory responses of human meningeal cells following challenge with Neisseria lactamica and with Neisseria meningitidis. Infection and immunity. 2006;74:6467-78.

[38] Wan T, Zhou X, Chen G, An H, Chen T, Zhang W, et al. Novel heat shock protein Hsp70L1 activates dendritic cells and acts as a Th1 polarizing adjuvant. Blood. 2004;103:1747-54.

[39] Saygili T, Akincilar SC, Akgul B, Nalbant A. Aggregatibacter actinomycetemcomitans GroEL protein promotes conversion of human CD4+ T cells into IFNgamma IL10 producing Tbet+ Th1 cells. PloS one. 2012;7:e49252.

[40] Rogers GL, Suzuki M, Zolotukhin I, Markusic DM, Morel LM, Lee B, et al. Unique roles of TLR9- and MyD88-dependent and -independent pathways in adaptive immune responses to AAV-mediated gene transfer. J Innate Immun. 2015.

[41] López-Yglesias AH, Zhao X, Quarles EK, Lai MA, VandenBos T, Strong RK, et al. Flagellin Induces Antibody Responses through a TLR5- and InflammasomeIndependent Pathway. The Journal of Immunology. 2014;192:1587-96. 


\section{FIGURE LEGENDS}

\section{Figure 1: Neisserial HSPCs induce TLR2-mediated signalling}

HEK293 cells expressing TLR2, or wildtype control HEK293 cells which lack this receptor, were cultured in media alone or stimulated for 24 hours with either $N$. lactamica (Nl) HSPC, N. meningitidis (MenB) HSPC, the TLR4 ligand LPS or the TLR2 ligand P3C ( $\mathrm{n}=6$ replica wells per stimulation). HEK293 TLR2 expression was confirmed by responsiveness to P3C. HSPCs from both Neisseria induced IL-8 secretion, only in cells expressing TLR2. ***p<0.001 compared to media control; ANOVA.

Figure 2: Role of immune cell TLR2 and TLR4 in the cytokine response to Neisserial HSPCs

Splenocytes from wildtype C57BL/6 $(\mathrm{n}=13), T \operatorname{Tr} 2^{-/}(\mathrm{n}=11)$ or $T l r 4^{0 / 0}$ mice $(\mathrm{n}=10)$ were cultured for 1 day either in media alone or with $N$. lactamica $(\mathrm{Nl})$ or $N$. meningitidis (MenB) HSPC. Controls were stimulated with P3C (TLR2 ligand) or LPS (TLR4 ligand). Cytokine levels in supernatants were determined by ELISA. As expected, $T l r 2^{-/}$splenocytes were non-responsive to $\mathrm{P} 3 \mathrm{C}$ and $\mathrm{Tlr} 4^{0 / 0}$ splenocytes were non-responsive to LPS. ${ }^{*} \mathrm{p}<0.05, * * \mathrm{p}<0.01, * * * \mathrm{p}<0.001$; significantly greater than media alone (ANOVA). 
Figure 3: Immune response induced by vaccination of mice with $N$. lactamica HSPC

a) The protein composition of $N$. lactamica HSPC was effectively unchanged by LPSreduction, when compared by SDS-PAGE. 1) N. lactamica lysate; 2) N. lactamica HSPC; 3) N. lactamica HSPC, LPS-reduced.

b-c) Wildtype C57BL/6 and $M y d 88^{-/}$mice (n=6) were vaccinated twice with $N$. lactamica HSPC or LPS-depleted N. lactamica HSPC ( $\downarrow$ LPS). Naïve controls $(n=6)$ were left unvaccinated. Sera and spleens were collected for analysis one week after

the second vaccination. b) Serum anti- $N$. lactamica and anti- $N$. meningitidis $\operatorname{IgG}$ levels were quantified by ELISA. Both vaccines increased antigen-specific antibody levels compared to naïve controls $(* * \mathrm{p}<0.01$, *** $\mathrm{p}<0.001$; ANOVA) but titres obtained with the LPS-reduced formulation were significantly lower (ANOVA). There was no difference in $\mathrm{IgG}$ levels between wildtype and $M y d 88^{-/}$mice. c) Splenocytes were cultured in media with or without $N$. lactamica lysate $(\mathrm{Nl})$ for 1 or 4 days, then cytokine levels in culture supernatants quantified by ELISA. Splenocytes from vaccinated wildtype but not $M y d 88^{-/}$mice secreted IFN $\gamma$ in response to $N$. lactamica lysate stimulation. $* * \mathrm{p}<0.01 ; * * * \mathrm{p}<0.001$ compared to unvaccinated control (ANOVA). n.s. = not significantly different (ANOVA).

Figure 4: Immune response induced by vaccination of mice with $N$. meningitidis type B HSPC

Wildtype C57BL/6 (n=8) and $M y d 88^{-/}$mice $(\mathrm{n}=7)$ were either left unvaccinated or vaccinated twice with $N$. meningitidis HSPC (MenB). Sera and spleens were collected for analysis one week after the second vaccination.

a) Serum anti- $N$. lactamica levels were quantified by ELISA. Both vaccines increased antigen-specific antibody levels compared to naïve controls (ANOVA). There was no difference in $\mathrm{IgG}$ levels between wildtype and $\mathrm{Myd}{ }^{-/}$mice.

b) Splenocytes were cultured in media with or without MenB HSPC for 1 or 4 days, then cytokine levels in culture supernatants quantified by ELISA. Splenocytes from wildtype but not $M y d 88^{-/}$mice secreted IFN $\gamma$ in response to MenB HSPC stimulation (ANOVA). There was no significant difference in the response of splenocytes from vaccinated or wildtype mice (ANOVA). 
Table 1 Proteomic analysis of Neisseria HspC vaccines

\begin{tabular}{|c|c|c|c|c|}
\hline & \multicolumn{2}{|l|}{ Neisseria lactamica $\mathrm{HspC}$} & \multicolumn{2}{|l|}{ Neisseria meningitidis $\mathrm{HspC}$} \\
\hline & Protein & $\% \mathrm{~mol}$ & Protein & $\% \mathrm{~mol}$ \\
\hline 1 & Elongation factor $\mathrm{Tu}$ & 7.110 & Oxidoreductase, zinc-binding dehydrogenase family protein & 6.271 \\
\hline 2 & Alcohol dehydrogenase & 5.456 & $(\mathrm{R}, \mathrm{R})$-butanediol dehydrogenase & 5.139 \\
\hline 3 & GroEL (Hsp60) & 3.306 & Elongation factor $\mathrm{G}$ & 2.837 \\
\hline 4 & 50S ribosomal protein $\mathrm{L} 4$ & 2.563 & Trigger factor 2 & 2.821 \\
\hline 5 & Universal stress protein & 2.459 & Phosphate acetyltransferase & 2.685 \\
\hline 6 & Elongation factor $\mathrm{G}$ & 2.344 & Glutamate dehydrogenase & 2.603 \\
\hline 7 & Peroxiredoxin 2 family protein/glutaredoxin & 2.278 & Antioxidant, AhpC/TSA family/glutaredoxin & 2.424 \\
\hline 8 & Ribosomal protein $\mathrm{S} 7$ & 1.992 & GroEL (Hsp60) & 2.405 \\
\hline 9 & Trigger factor & 1.975 & Elongation factor Ts & 2.340 \\
\hline 10 & $30 \mathrm{~S}$ ribosomal protein $\mathrm{S} 9$ & 1.942 & Acetate kinase 1 & 2.335 \\
\hline 11 & $30 \mathrm{~S}$ ribosomal protein $\mathrm{S} 1$ & 1.876 & 30S ribosomal protein $\mathrm{S} 1$ & 2.144 \\
\hline 12 & Homoprotocatechuate degradation operon regulator & 1.805 & NifU-like protein & 2.097 \\
\hline 13 & 50S ribosomal protein $\mathrm{L} 10$ & 1.794 & 5-methyltetrahydropteroyltriglutamate-homocysteine methyltransferase & 2.017 \\
\hline 14 & Identified by MetaGeneAnnotator & 1.726 & Oligopeptidase A & 1.981 \\
\hline 15 & Succinyl-CoA ligase [ADP-forming] subunit beta & 1.689 & Succinyl-CoA ligase [ADP-forming] subunit beta & 1.978 \\
\hline 16 & NifU-like protein & 1.670 & Ribosomal protein S7 & 1.815 \\
\hline 17 & 50S ribosomal protein L16 & 1.657 & Phosphoglycerate kinase & 1.645 \\
\hline 18 & Phosphate acetyltransferase & 1.653 & Cell division protein $\mathrm{ftsZ}$ & 1.637 \\
\hline 19 & Glyceraldehyde 3-phosphate dehydrogenase & 1.630 & DnaK (Hsp70) & 1.605 \\
\hline 20 & DnaK (Hsp70) & 1.629 & ATP synthase subunit beta & 1.595 \\
\hline
\end{tabular}




\section{Figure 1}
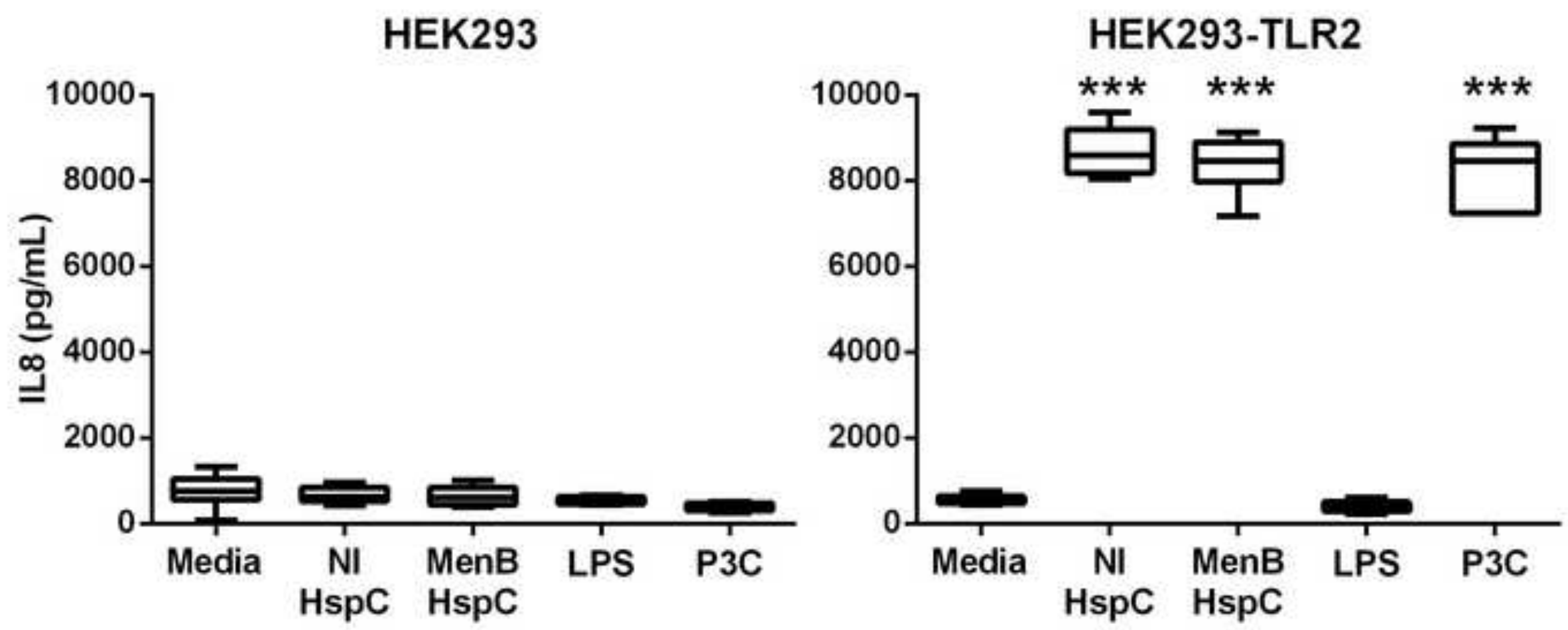
Figure 2
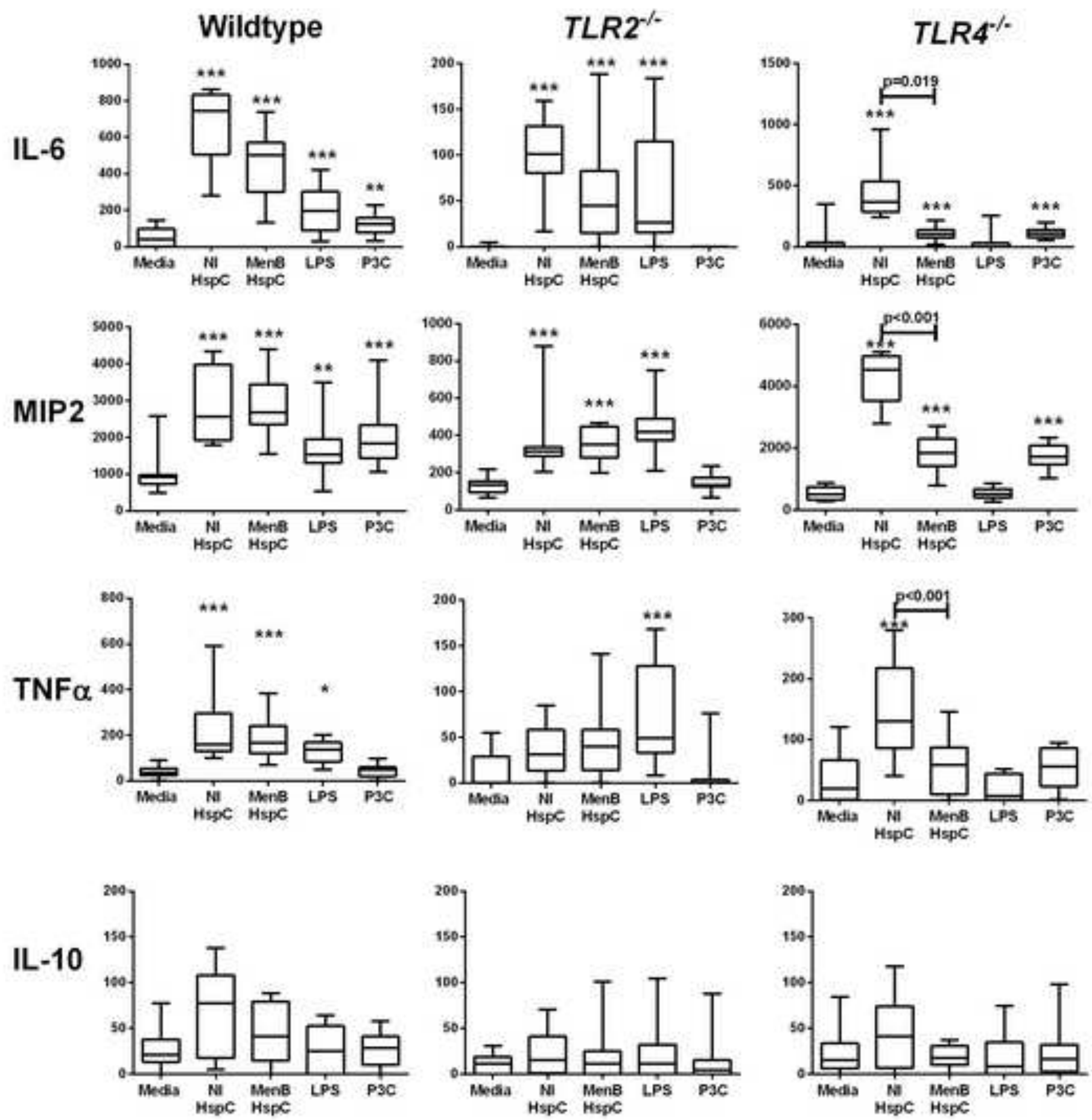
Figure 3

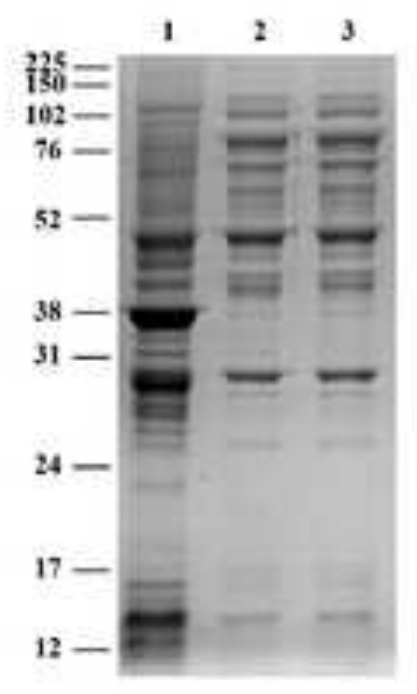

a)
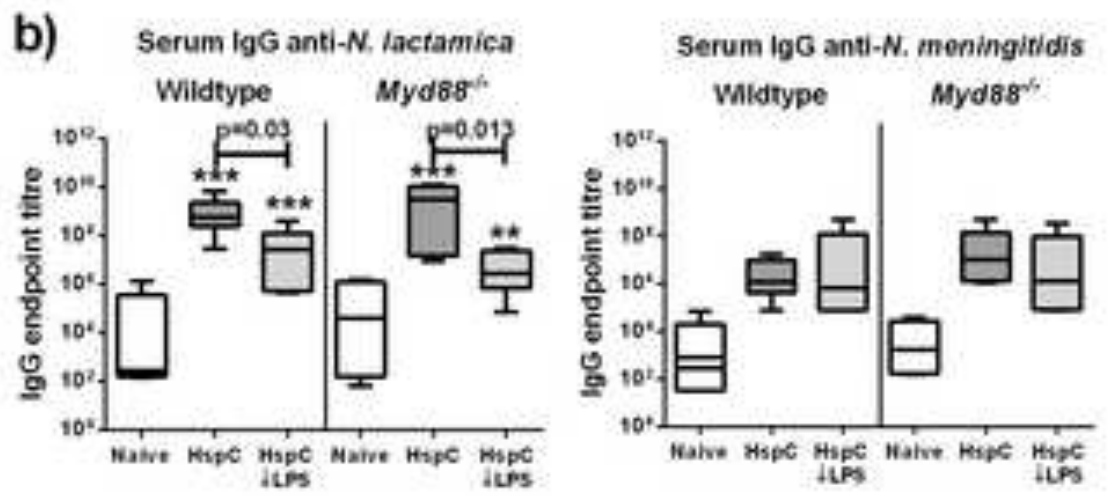

c)

1 day stimulation

4 days stimulation

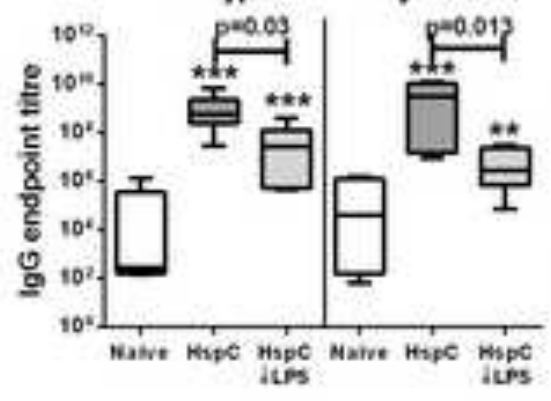

trs
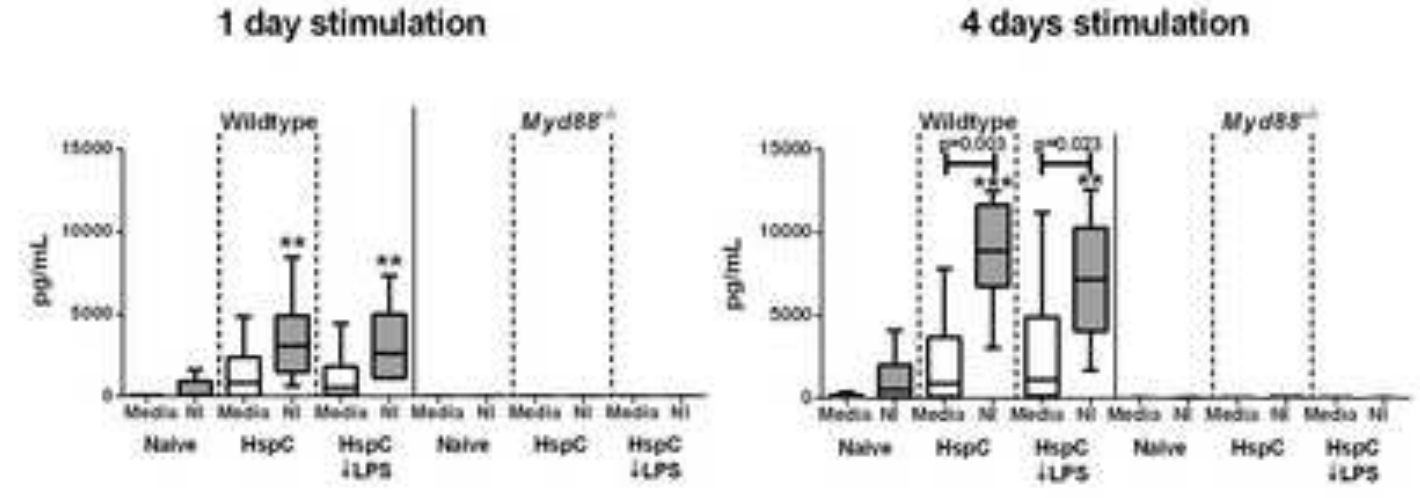

IFNy
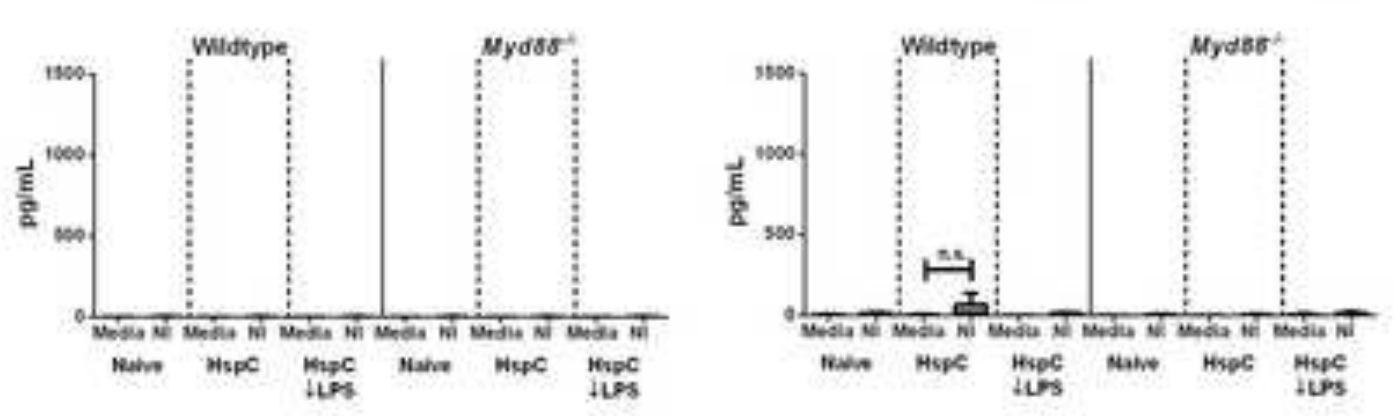

IL -13
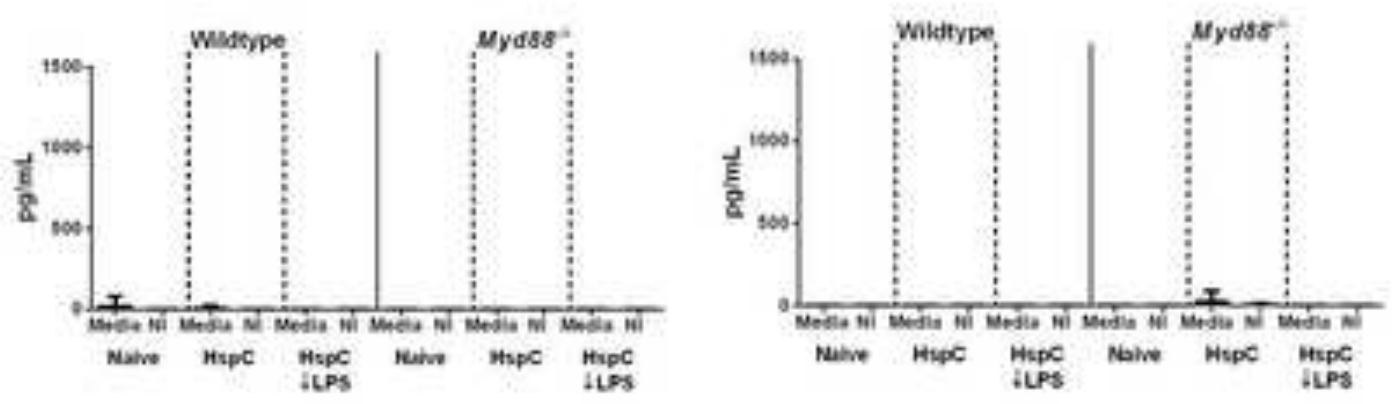
Figure 4

a)

Serum IgG1

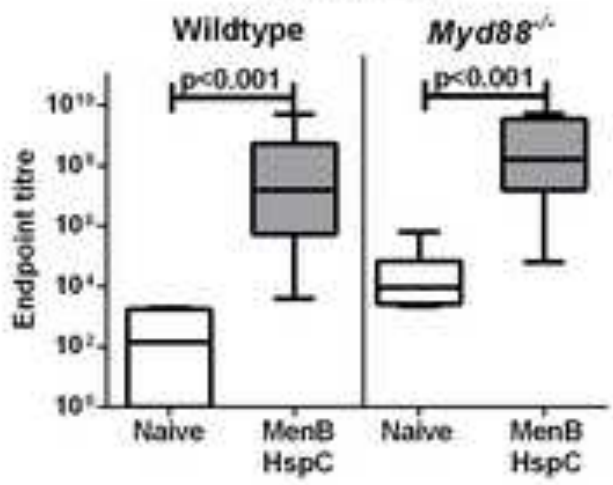

b)
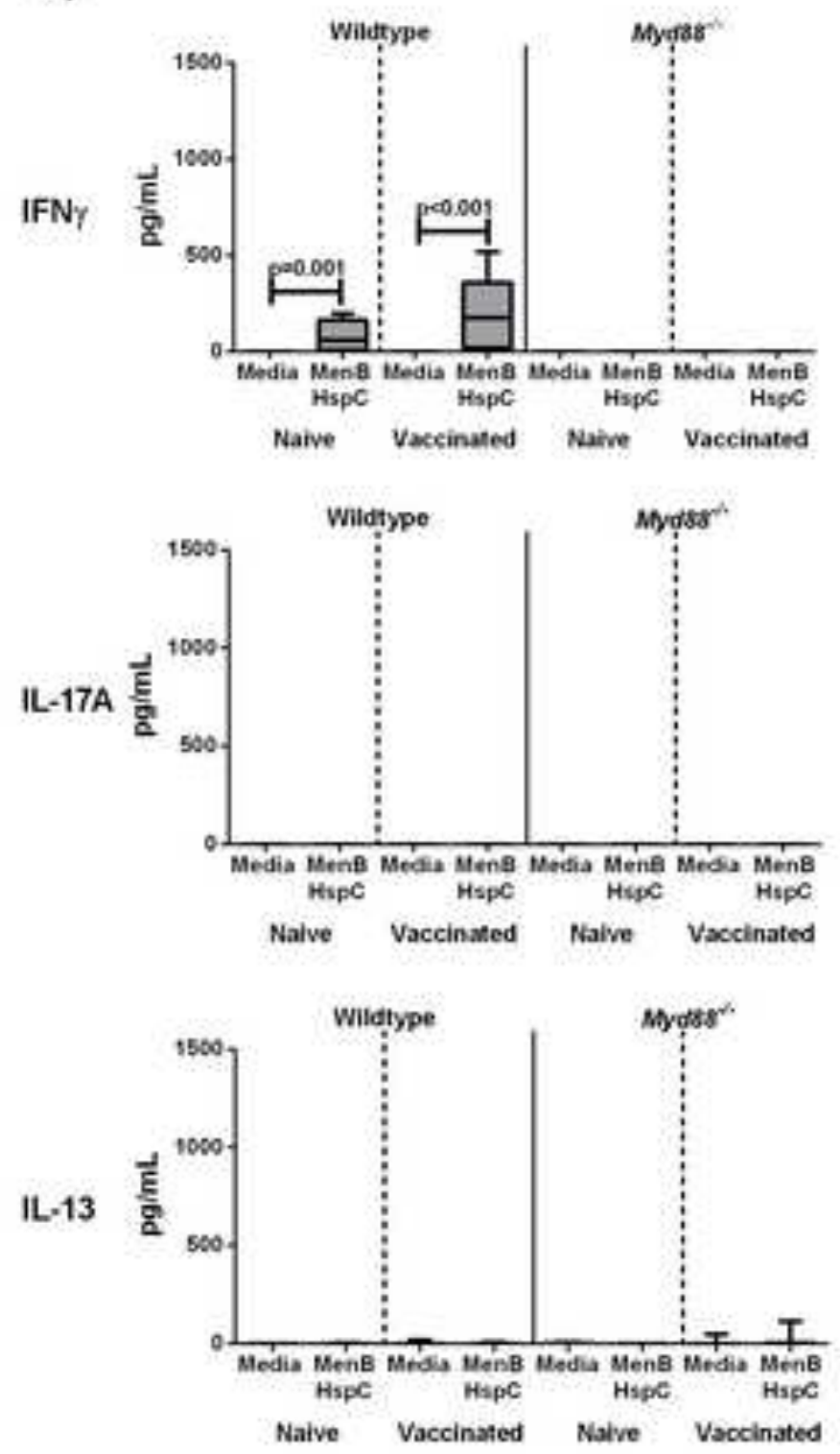
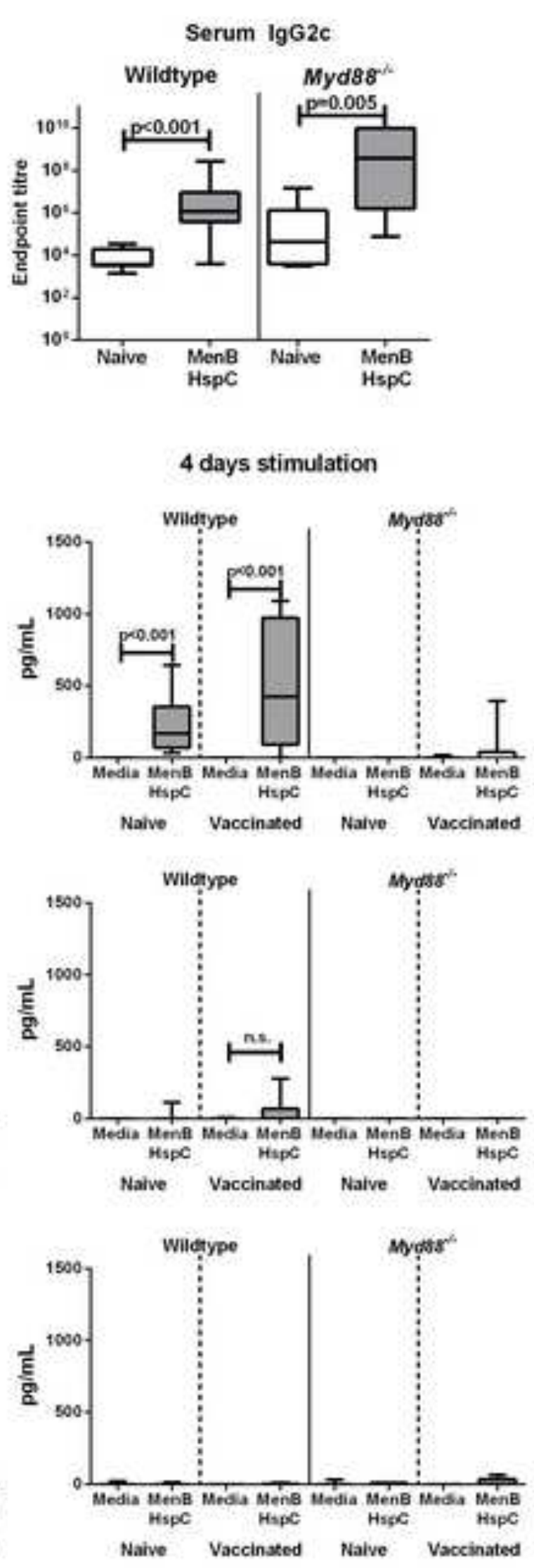